\title{
Conventional Surfactants and a Model Based on Molecular Descriptors as Alternatives to the Drug Solubility in Fasted State Simulated Intestinal Fluid
}

\author{
Nahal Rahimi Ardabili ${ }^{1}$ and Ali Shayanfar ${ }^{2,3^{*}}$ \\ ${ }^{1}$ Student Research Committee, Faculty of Pharmacy, Tabriz University of Medical Sciences, Tabriz, Iran. \\ ${ }^{2}$ Pharmaceutical Analysis Research Center, Tabriz University of Medical Science, Tabriz, Iran. \\ ${ }^{3}$ Department of Pharmaceutical Chemistry, Faculty of Pharmacy, Tabriz University of Medical Sciences, Tabriz, Iran.
}

e-mail: shayanfara@tbzmed.ac.ir

\begin{abstract}
Biorelevant media, such as fasted state simulated intestinal fluid (FaSSIF), are often used to forecast in vivo behavior of oral solid formulations in the gastrointestinal tract. This study uses conventional surfactants (Tween 80 and sodium lauryl sulfate) and a model based on structural parameters (i.e., molecular descriptors) to evaluate possible alternatives to FaSSIF for solubility evaluation of three poorly soluble drugs. The solubility of enrofloxacin, lamotrigine, and phenobarbital was determined in phosphate buffer solution (PBS, pH 6.5) and FaSSIF. The molecular descriptors of drugs were computed, and a mathematical model based on logistic regression was generated to estimate solubilization in FaSSIF compared with PBS. The results demonstrated that solubility of enrofloxacin, lamotrigine, and phenobarbital in Tween $80(0.1 \% \mathrm{w} / \mathrm{v})$ was similar to their solubility in FaSSIF, and the proposed model calculated the solubilization ratio with $80 \%$ accuracy. In conclusion, Tween 80 in low concentration (i.e., $0.05 \%$ ) is an appropriate low-cost alternative to FaSSIF, and the proposed model can be used to evaluate the possibility of solubilization in FaSSIF.
\end{abstract}

KEYWORDS: Biorelevant media, modeling, solubility, structural parameters, surfactant, dissolution

\section{INTRODUCTION}

$\mathrm{O}$ ral dosage forms are advantageous and easy to take, so they are the most common way of drug administration and have high patient compliance. Solubility is one of the most important physicochemical properties of pharmaceuticals. In vitro tests for solubility and dissolution of drug candidates and formulations are used to estimate oral bioavailability in the drug discovery and development phase before conducting timeconsuming and expensive in vivo tests and clinical trials (1-4). The dissolution rate is a commonly used in vitro assessment based on the Noyes-Whitney equation, and thermodynamic solubility has a substantial effect on the dissolution rate. Moreover, some in vivo preclinical studies that use animal species (rat and dog) for evaluation of oral bioavailability have not been recommended because of the lack of reasonable in vitro-in vivo correlation (5).

Various strategies for simulation of the gastrointestinal tract (GI) in humans have been proposed. Hydrochloric acid $(\mathrm{HCl})$ and phosphate buffer solutions (PBS) are the most common media that have been recommended for testing the release of drugs in United States Pharmacopoeia (USP) $(6,7)$. However, the GI tract is complex, and the measured solubility and dissolution rate in $\mathrm{HCl}$ and PBS are not always indicative of oral bioavailability (8).

Recently, the development of biorelevant media including fasted state simulated intestinal fluid (FaSSIF) and fed state simulated intestinal fluid (FeSSIF) have played an essential role in simulating the $\mathrm{Gl}$ environment (9). These media can be used to evaluate the dissolution and solubility of oral dosing forms. They contain a surfactant (sodium taurocholate) and lecithin (phospholipid), which are expensive and can restrict their routine application, so there is a need to identify alternative media to overcome the cost barrier. For example, media containing conventional surfactants such as sodium lauryl sulfate (SLS) and Tween 80 have a low cost and some evidence has shown that drug solubility in these media is similar to biorelevant media $(10,11)$. Therefore, modeling the relation between structural descriptors 
and the physicochemical and pharmacokinetic activity of biological compounds has been used to predict a property of a drug or drug candidate without any in vitro and in vivo studies $(8,12,13)$. It can significantly decrease the cost of developing a drug or formulation.

In this study, three poorly soluble drugs, i.e., an acidic (phenobarbital), a zwitterion (enrofloxacin) and a basic drug (lamotrigine) were selected. Their chemical structures are illustrated in Figure 1. Based on USP and aqueous solubility values reported in the literature, enrofloxacin, lamotrigine, and phenobarbital drugs are very slightly soluble (1000-10,000 parts solvent required for dissolving 1 part solute) $(6,14,15)$. According to the Biopharmaceutics Classification System (BCS), enrofloxacin and lamotrigine belong to class II and phenobarbital is a class I drug (16-18).

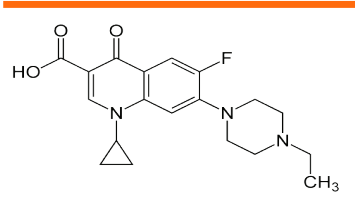

A

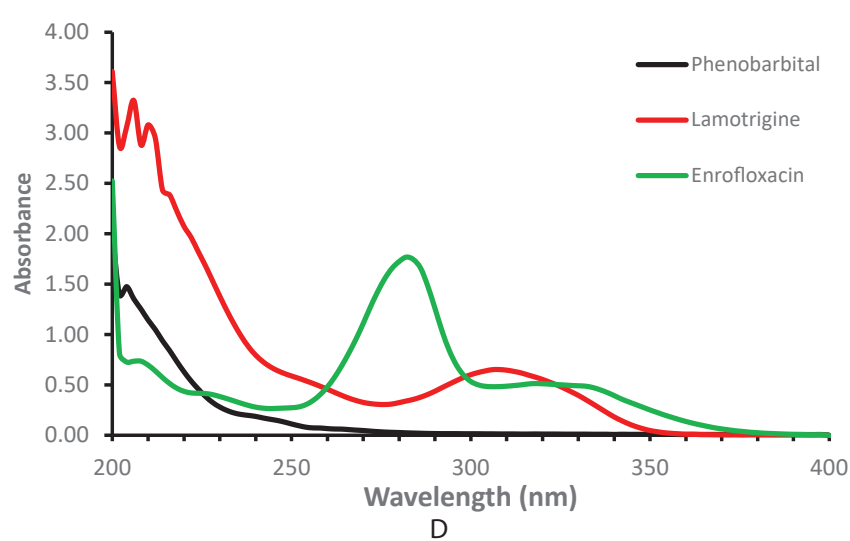

Figure 1. Structure of enrofloxacin (A), lamotrigine (B), phenobarbital (C), and their ultraviolet spectra (D).

To our knowledge, there are no reports about solubility of enrofloxacin, lamotrigine, and phenobarbital in FaSSIF. This study aims to determine experimental solubility of drugs in PBS ( $\mathrm{pH}$ 6.5) and FaSSIF and to investigate the use of conventional surfactants (i.e., SLS and Tween 80 ) as a low-cost alternative. A logistic regression model based on structural descriptors was developed to predict the solubility of drugs in PBS, FaSSIF, Tween 80 , and SLS.

\section{MATERIALS AND METHODS}

\section{Materials}

Enrofloxacin was purchased from Temad Company (Iran) and lamotrigine was obtained from Arastoo Pharmaceutical Company (Iran). Phenobarbital was a gift from Pars Darou Company (Iran), and sodium hydroxide, ethanol, and dichloromethane were provided from Merck (Germany). For the preparation of PBS, sodium chloride and sodium dihydrogen phosphate were supplied from the Scharlau (Spain). Sodium taurocholate was purchased from ACROS Organics (USA), and soya lecithin was provided from Lipoid Company (Germany) to prepare of FaSSIF. Lab-made distilled water was used as a solvent in this study. Tween 80 and SLS were applied as surfactants and purchased from Merck.

\section{Preparation of PBS}

To prepare PBS, $323 \mathrm{mg}$ of sodium dihydrogen phosphate, $61 \mathrm{mg}$ of sodium chloride, and $40 \mathrm{mg}$ of sodium hydroxide were used. The $\mathrm{pH}$ of the buffer was adjusted to 6.5 with a $1 \mathrm{M}$ sodium hydroxide solution (10). Three concentrations of PBS $(0.05,0.1$, and $0.5 \% \mathrm{w} / \mathrm{v})$ were prepared from dissolving an appropriate amount of Tween 80 and SLS in PBS.

\section{Preparation of FaSSIF}

The FaSSIF medium was made based on a previously published method (10). Briefly, $300 \mu \mathrm{L}$ of $50-\mathrm{mg}$ lecithin was dissolved in $0.5 \mathrm{~mL}$ dichloromethane and added to PBS, and an emulsion solution was formed. For complete evaporation of dichloromethane, the solution was placed on a hot plate for 45 minutes at $50^{\circ} \mathrm{C}$. It was cooled after the complete removal of dichloromethane and clarification of the solution. Subsequently, $83 \mathrm{mg}$ of sodium taurocholate was added to the solution. Distilled water was added to reach a final volume of $50 \mathrm{~mL}$.

\section{Solubility Determination}

The solubility of enrofloxacin, lamotrigine, and phenobarbital was studied in PBS, FaSSIF, Tween 80, SLS at various concentrations. An excess amount of drug powder was added into a certain volume of different media, and they were placed inside the shaker-incubator (Heidolph, Germany) at $37{ }^{\circ} \mathrm{C}$ and $150 \mathrm{rpm}$ for 72 hours. Next, the solutions were filtered with a $0.22-\mu \mathrm{m}$ hydrophilic filter (Durapore, Millipore, Ireland) and diluted with an appropriate amount of ethanol. The ultraviolet (UV) absorption of each solution was measured by spectrophotometry (Shimadzu, Japan) at 280, 307, and $220 \mathrm{~nm}$ for enrofloxacin, lamotrigine and phenobarbital, respectively (Fig. 1D). The concentrations were determined based on the plotted calibration curve for each drug. The experimental data were reported as mean \pm SD for at least three replicates. UV spectrophotometry is an appropriate analysis method for evaluation of solubility in FaSSIF whenever absorption of solute is above 300 $\mathrm{nm}$ (lamotrigine) and also below $300 \mathrm{~nm}$ if the saturated solution has to be diluted at least 100 times (saturated 
solutions of enrofloxacin and phenobarbital were diluted more than 100 fold to maintain linearity according to the calibration curve) (19).

\section{Computational Methods}

Previously reported solubility data of 74 drugs in PBS and FaSSIF were collected from the literature (referred to as "experimental" SR) (19-21). The solubilization ratio (SR) in FaSSIF compared with PBS (logarithm unit) was calculated (referred to as "calculated" SR), and the data were classified into two groups:

- Group 1: SR > 1.1, FaSSIF has a considerable effect on solubility.

- Group 2: SR <1.1, FaSSIF has no considerable effect on solubility.

The structural parameters (i.e., molecular descriptors) of drugs in the proposed model included: Abraham solvation parameters ( $e$ : excess molar refraction, $S$ : polarity/ polarizability descriptors of the solute, $A$ and $B$ : the solute hydrogen-bond acidity and basicity, respectively, and $V$ : McGowan volume); molecular weight; melting point; number of rotatable bonds; topological polar surface area (TPSA); partition coefficient (log P); and distribution coefficient at pH $6.5\left(\log \mathrm{D}_{6.5}\right)$. These were was computed with ACD-I-Lab software (https://ilab.acdlabs.com). Logistic regression was performed using SPSS 17 (IBM) to develop a model for classification of drugs based on the SR $(22,23)$. The experimental SR (Group 1 or 2 ) was used as a dependent parameter and structural descriptors of the solute were used as independent parameters to develop the model. Thus, a model was developed to predict the drug's group.

\section{RESULTS AND DISCUSSION}

\section{Solubility of Studied Drugs in FaSSIF and Surfactant Solutions}

Table 1 shows the solubility of enrofloxacin, lamotrigine, and phenobarbital in PBS, FaSSIF, SLS and Tween 80 at different concentrations. Solubility in FaSSIF in comparison with PBS was not considerably changed (difference in solubility values were $<30 \%$ ). Moreover, solubility of thestudied drugs did not increasesubstantially (<30\%) in SLS 0.05\% and 0.1\% (except lamotrigine) and Tween 80 in all of the studied concentrations (except lamotrigine in $0.1 \%$ and $0.5 \%$ ).

An effective mechanism for solubilization by surfactants is the formation of micelles, and all of the drugs had an increase in solubility in $0.5 \% \mathrm{SLS}$, which has a critical micelle concentration (CMC) of $0.24 \%$. It could be related to the hydrophilic and low hydrogen bond donor functional groups (Fig. 1), which have positive and negative effects on solubilization by surfactant, respectively (25). The studied concentration of Tween 80 was higher than its CMC (0.002\%) (24); however, a slight or unremarkable increase was observed in the solubility values (0.5\%).

These data confirmed that the solubilization power of Tween 80 greatly depends on lipophilicity of the solute (the studied drugs are relatively hydrophilic compounds $\left.\log D_{6.5}<1.7\right)$, and its concentration. A solubilityenhancing effect has been observed at concentrations higher than $1 \%$ (26).

The SR of the three drugs in Tween 80 and SLS compared with FaSSIF is illustrated in Figure 2. It shows a more similar pattern between Tween 80 (in low concentration) and FaSSIF against of SLS. These results confirm previous reports that Tween 80 in low concentration (i.e., 0.05\%) is an appropriate alternative medium for evaluating solubility in FaSSIF (8).

\section{Modeling Drug Solubilization in FaSSIF}

The solubility data in PBS and FaSSIF (log S and SR), molecular descriptors of the drugs, and the experimental and calculated groups were listed in Table 2. According to logistic regression, Equation 1 shows the proposed model for evaluating the probability of increasing drug solubility in FaSSIF compared to PBS.

Table 1. Solubility Data of Enrofloxacin, Lamotrigine, and Phenobarbital in PBS, FaSSIF, Tween 80, and SLS at $37^{\circ} \mathrm{C}$

\begin{tabular}{|c|c|c|c|c|c|c|c|c|}
\hline & \multirow{2}{*}{ PBS } & \multirow{2}{*}{ FaSSIF } & \multicolumn{3}{|c|}{ Tween 80 } & \multicolumn{3}{c|}{ SLS } \\
\cline { 5 - 9 } & & & $\mathbf{0 . 0 5 \%}$ & $\mathbf{0 . 1 \%}$ & $\mathbf{0 . 5 \%}$ & $\mathbf{0 . 0 5 \%}$ & $\mathbf{0 . 1 \%}$ & $\mathbf{0 . 5 \%}$ \\
\hline Enrofloxacin & $0.504 \pm 0.030$ & $0.500 \pm 0.028$ & $0.535 \pm 0.022$ & $0.530 \pm 0.020$ & $0.529 \pm 0.018$ & $0.514 \pm 0.022$ & $0.546 \pm 0.011$ & $1.497 \pm 0.094$ \\
\hline Lamotrigine & $0.313 \pm 0.034$ & $0.399 \pm 0.038$ & $0.394 \pm 0.014$ & $0.576 \pm 0.006$ & $0.603 \pm 0.013$ & $0.487 \pm 0.011$ & $0.512 \pm 0.008$ & $1.074 \pm 0.014$ \\
\hline Phenobarbital & $2.110 \pm 0.091$ & $2.062 \pm 0.163$ & $1.902 \pm 0.188$ & $2.095 \pm 0.092$ & $2.403 \pm 0.087$ & $2.186 \pm 0.036$ & $2.323 \pm 0.087$ & $2.910 \pm 0.190$ \\
\hline
\end{tabular}

Data are presented as mean $\pm S D$ in $\mathrm{g} / \mathrm{L}$.

PBS: phosphate buffer solution ( $\mathrm{pH}=6.5$ ), FaSSIF: fasted state simulated intestinal fluid, SLS: sodium lauryl sulfate 


$$
p=\frac{e^{\left(3.963-0.925 \log D_{6.5}-0.014 T P S A-1.652 V+2.290 S\right)}}{1+e^{\left(3.963-0.925 \log D_{6.5}-0.014 T P S A-1.652 V+2.290 S\right)}}
$$

In this model, $p$ is probability of a binary response (class 1: $p<0.5$ or 2: $p>0.5$ ) based on $\log D_{6}$, TPSA is topological polar surface, $S$ is dipolarity/polarizability descriptor, $V$ is McGowan volume, and $e$ is Euler's number $(e=2.718$ ) (2729). Probability values $(p)$ associated with each selected descriptor were less than 0.1. The most important factor in predicting the increased drug solubility in FaSSIF compared to PBS is $\log D_{6.5}$. The average $\log \mathrm{D}_{6.5}$ value for Group 1 (SR > 1.1) and 2 (SR < 1.1) are $4.00 \pm 1.69$ and $2.38 \pm 1.39$, respectively, and this difference is statistically significant $(p<0.001)$. In Group 1 (experimental data), $79 \%(23 / 29)$ have a $\log \mathrm{D}_{6.5}$ greater than 3 compared to $29 \%(13 / 45)$ in Group 2. This result indicates that the probability of increasing drug solubility in FaSSIF is higher for lipophilic drugs. For most lipophilic drugs, there is a considerable difference between solubility in PBS and FaSSIF. Sodium taurocholate is another example of a surfactant in which solubilization is observed for lipophilic compounds (19). A similar pattern has been reported for drug solubilization in SLS (25). In addition to $\log D_{6.5}$, TPSA, $V$, and $S$ have a substantial effect on the prediction accuracy of the proposed model. Given the relationship between molecular descriptors, especially $\log D_{6.5}$ and solubilization in FaSSIF, the proposed model can be used to evaluate the possibility of solubilization in this medium. The proposed model can calculate the experimental SR group with approximately $80 \%$ (59/74) accuracy $(69 \%$ and $87 \%$ for Group 1 and 2, respectively.)

Enrofloxacin, lamotrigine, and phenobarbital are relatively hydrophilic drugs $\left(\log \mathrm{D}_{6.5}<1.7\right)$, and their solubility did not considerably increase in FaSSIF compared with PBS (Group 2).

\section{CONCLUSION}

The solubility of enrofloxacin, lamotrigine, and phenobarbital in FaSSIF, compared with PBS, did not increase similar to solubility in the low concentration of Tween 80. Therefore, it can be used as an alternative biorelevant media for solubility tests. Moreover, the proposed model based on molecular descriptors predicted the effect of FaSSIF on drug solubility with good accuracy.

\section{FUNDING}

This work was financially supported by Tabriz University of Medical Sciences (no. 63148).

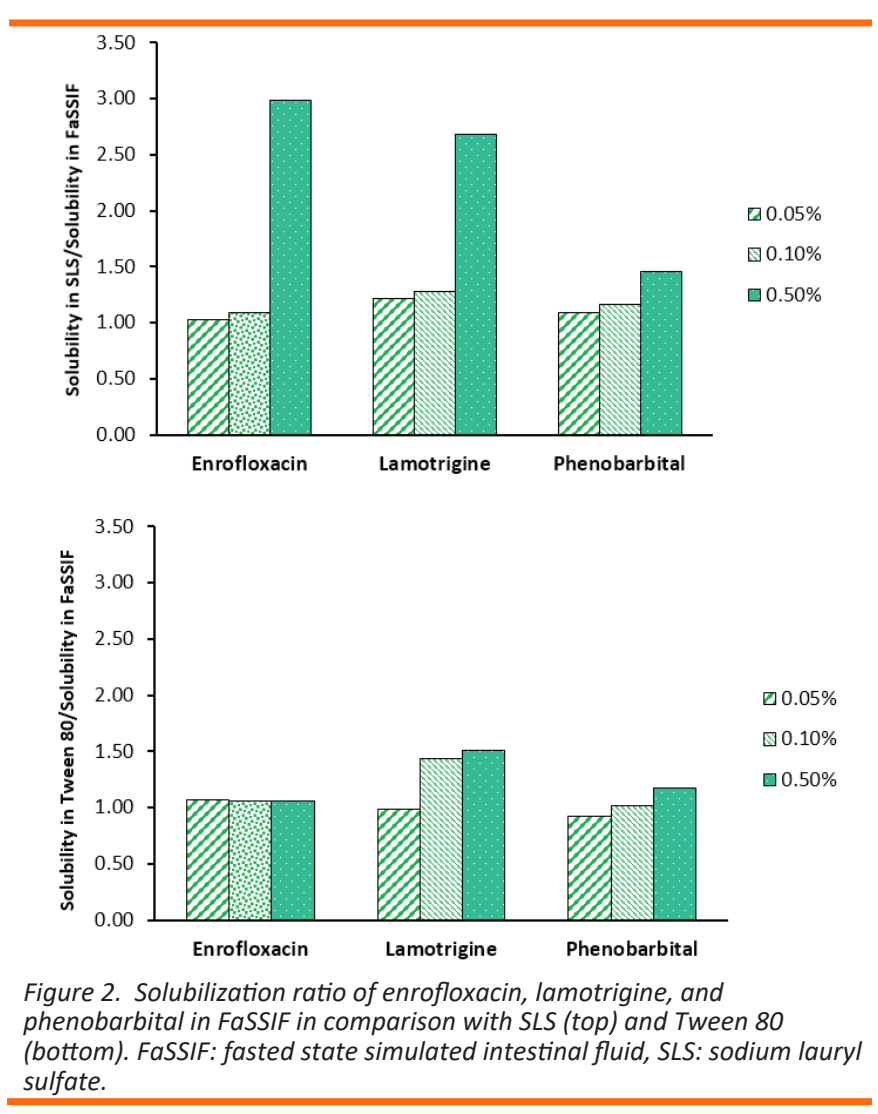

\section{CONFLICTS OF INTEREST}

The authors disclosed no conflicts of interest related to this article.

\section{REFERENCES}

1. Martínez F.; Jouyban, A.; Acree, W. E. Pharmaceuticals solubility is still nowadays widely studied everywhere. Pharm. Sci. 2017, 23, 1-2. DOI: 10.15171/PS.2017.01.

2. Reppas C.; Vertzoni, M. Biorelevant in-vitro performance testing of orally administered dosage forms. J Pharm Pharmacol. 2012, 64, 919-930. DOI: 10.1111/j.20427158.2012.01474.x.

3. Klein S. The use of biorelevant dissolution media to forecast the in vivo performance of a drug. AAPS J. 2010, 12, 397-406. DOI: 10.1208/s12248-010-9203-3.

4. Yu J.; Wu, N.; Zheng, X.; Zheng, M. Improvement of bioavailability and dissolution of tanshinone iia by encapsulating it with hydroxypropyl- $\beta$-cyclodextrin. Pharm. Sci. 2020, 26, 296-305. DOI: 10.34172/PS.2020.31.

5. Musther H.; Olivares-Morales, A.; Hatley, O. J. D.; Liu, B.; Rostami Hodjegan, A. Animal versus human oral drug bioavailability: do they correlate? Eur. J. Pharm. Sci. 2014, 57, 280-291. DOI: 10.1016/j.ejps.2013.08.018.

6. $\quad<788>$ Particulate Matter in Injections. The United States Pharmacopeia and National Formulary USP 41-NF 36; The United States Pharmacopeial Convention, Inc.: Rockville, MD, 2018. 
Table 2. Solubility Data and Molecular Descriptors of Drugs Including Experimental and Calculated Group

\begin{tabular}{|c|c|c|c|c|c|c|c|c|c|c|c|c|}
\hline \multirow[b]{2}{*}{ Ref } & \multirow[b]{2}{*}{ Drug } & \multicolumn{3}{|c|}{ Solubility (log S) } & \multicolumn{4}{|c|}{ Molecular Descriptors } & \multicolumn{2}{|c|}{ SR Group* } & \multirow[b]{2}{*}{$p$} & \multirow{2}{*}{$\begin{array}{l}\text { Prediction } \\
\text { result }\end{array}$} \\
\hline & & PBS (M) & FaSSIF (M) & $\mathrm{SR}^{*}$ & $\log \mathrm{D}_{6.5}$ & TPSA & $s$ & $v$ & Exp & Calc & & \\
\hline (20) & Probucol & -8.94 & -5.18 & 1.73 & 10.00 & 14.9 & 1.38 & 4.45 & 1 & 1 & 0.00 & $\sqrt{ }$ \\
\hline (20) & Tipranavir & -5.19 & -4.47 & 1.16 & 6.00 & 189.0 & 3.08 & 4.28 & 1 & 1 & 0.01 & $\sqrt{ }$ \\
\hline$(20)$ & Rimonabant & -6.39 & -4.62 & 1.38 & 5.30 & 227.0 & 3.13 & 3.21 & 1 & 1 & 0.09 & $\sqrt{ }$ \\
\hline$(20)$ & Fenofibrate & -6.26 & -4.58 & 1.37 & 5.30 & 138.0 & 2.11 & 2.72 & 1 & 1 & 0.07 & $\sqrt{ }$ \\
\hline$(20)$ & Amiodarone & -7.30 & -3.26 & 2.24 & 5.20 & 193.0 & 2.49 & 3.75 & 1 & 1 & 0.02 & $\sqrt{ }$ \\
\hline (20) & Felodipine & -5.51 & -3.85 & 1.43 & 4.80 & 136.0 & 1.85 & 2.71 & 1 & 1 & 0.06 & $\sqrt{ }$ \\
\hline$(20)$ & Tamoxifen & -4.80 & -3.38 & 1.42 & 4.80 & 8.6 & 1.85 & 3.17 & 1 & 1 & 0.17 & $\sqrt{ }$ \\
\hline$(20)$ & Diethylstilbestrol & -4.31 & -3.83 & 1.13 & 4.80 & 80.2 & 1.53 & 2.24 & 1 & 1 & 0.14 & $\sqrt{ }$ \\
\hline$(20)$ & Nelfinavir & -6.16 & -3.68 & 1.67 & 4.70 & 83.8 & 3.62 & 4.54 & 1 & 1 & 0.31 & $\sqrt{ }$ \\
\hline$(20)$ & Ivermectin & -6.10 & -3.86 & 1.58 & 4.70 & 112.0 & 3.21 & 6.72 & 1 & 1 & 0.00 & $\checkmark$ \\
\hline$(20)$ & Aprepitant & -6.16 & -4.37 & 1.41 & 4.40 & 325.0 & 2.17 & 3.27 & 1 & 1 & 0.01 & $\sqrt{ }$ \\
\hline$(20)$ & Astemizole & -4.38 & -3.67 & 1.19 & 4.40 & 57.6 & 2.70 & 3.56 & 1 & 1 & 0.34 & $\sqrt{ }$ \\
\hline$(20)$ & Cinnarizine & -5.42 & -4.44 & 1.22 & 4.30 & 2.6 & 2.12 & 3.11 & 1 & 1 & 0.42 & $\sqrt{ }$ \\
\hline (20) & Lopinavir & -5.76 & -4.04 & 1.43 & 4.20 & 67.0 & 4.57 & 5.06 & 1 & 2 & 0.77 & $x$ \\
\hline (20) & Ketoconazole & -4.56 & -3.50 & 1.30 & 3.90 & 181.0 & 3.76 & 3.72 & 1 & 2 & 0.55 & $x$ \\
\hline$(20)$ & Gefitinib & -5.04 & -3.72 & 1.35 & 3.80 & 147.0 & 2.97 & 3.15 & 1 & 1 & 0.48 & $\sqrt{ }$ \\
\hline (20) & Atovaquone & -5.93 & -5.29 & 1.12 & 3.70 & 155.0 & 2.54 & 2.69 & 1 & 1 & 0.42 & $\sqrt{ }$ \\
\hline$(20)$ & Danazol & -5.75 & -4.60 & 1.25 & 3.60 & 68.8 & 2.38 & 2.67 & 1 & 2 & 0.66 & $x$ \\
\hline$(20)$ & Terfenadine & -4.62 & -3.74 & 1.24 & 3.50 & 46.2 & 2.04 & 4.01 & 1 & 1 & 0.13 & $\sqrt{ }$ \\
\hline (20) & Nitrendipine & -4.95 & -4.35 & 1.14 & 3.50 & 126.0 & 2.26 & 2.64 & 1 & 1 & 0.43 & $\checkmark$ \\
\hline$(20)$ & Pranlukast & -5.17 & -3.75 & 1.38 & 3.30 & 177.0 & 4.10 & 3.50 & 1 & 2 & 0.88 & $x$ \\
\hline$(20)$ & Loviride & -5.55 & -4.92 & 1.13 & 3.20 & 177.0 & 2.59 & 2.50 & 1 & 2 & 0.56 & $x$ \\
\hline$(20)$ & Rifampicin & -2.92 & -2.61 & 1.12 & 3.00 & 158.0 & 4.67 & 6.21 & 1 & 1 & 0.34 & $\sqrt{ }$ \\
\hline$(20)$ & Phenazopyridine & -3.08 & -2.67 & 1.15 & 2.80 & 115.0 & 1.67 & 1.64 & 1 & 2 & 0.69 & $x$ \\
\hline$(20)$ & Lorazepam & -3.44 & -2.93 & 1.17 & 2.60 & 213.0 & 1.83 & 2.11 & 1 & 1 & 0.30 & $\checkmark$ \\
\hline$(20)$ & Flufenamic & -2.75 & -2.48 & 1.11 & 2.60 & 167.0 & 1.36 & 1.83 & 1 & 1 & 0.32 & $\checkmark$ \\
\hline (20) & Nevirapine & -3.51 & -3.14 & 1.12 & 1.70 & 55.9 & 2.29 & 1.94 & 1 & 2 & 0.97 & $x$ \\
\hline$(20)$ & Naproxen & -3.00 & -2.67 & 1.12 & 1.30 & 84.5 & 1.49 & 1.78 & 1 & 2 & 0.88 & $x$ \\
\hline$(20)$ & Indoprofen & -2.98 & -2.66 & 1.12 & 0.70 & 102.0 & 2.30 & 2.11 & 1 & 2 & 0.97 & $x$ \\
\hline$(21)$ & Sulindac & -2.82 & -2.66 & 1.06 & 6.32 & 73.6 & 2.72 & 2.57 & 2 & 1 & 0.27 & $x$ \\
\hline (20) & Clotrimazole & -5.17 & -5.00 & 1.03 & 5.20 & 50.4 & 2.37 & 2.62 & 2 & 1 & 0.38 & $x$ \\
\hline$(20)$ & Ritonavir & -5.27 & -5.07 & 1.04 & 4.60 & 77.9 & 5.05 & 5.55 & 2 & 2 & 0.73 & $\sqrt{ }$ \\
\hline (20) & Saquinavir & -3.93 & -3.57 & 1.10 & 4.10 & 152.0 & 5.55 & 5.30 & 2 & 2 & 0.87 & $\sqrt{ }$ \\
\hline (20) & Irbesartan & -3.62 & -3.58 & 1.01 & 4.00 & 108.0 & 2.71 & 3.32 & 2 & 1 & 0.36 & $x$ \\
\hline$(20)$ & Loperamide & -4.04 & -3.67 & 1.10 & 3.90 & 112.0 & 2.90 & 3.77 & 2 & 1 & 0.30 & $x$ \\
\hline$(20)$ & Glibenclamide & -5.04 & -5.02 & 1.00 & 3.90 & 203.0 & 3.84 & 3.56 & 2 & 2 & 0.58 & $\sqrt{ }$ \\
\hline (20) & Progesterone & -4.45 & -4.09 & 1.09 & 3.80 & 63.1 & 2.49 & 2.62 & 2 & 2 & 0.71 & $\sqrt{ }$ \\
\hline (19) & Papaverine & -4.07 & -3.79 & 1.07 & 3.52 & 49.8 & 2.76 & 2.59 & 2 & 2 & 0.88 & $\sqrt{ }$ \\
\hline$(21)$ & Albendazole & -5.49 & -5.14 & 1.07 & 3.20 & 79.7 & 1.96 & 1.95 & 2 & 2 & 0.75 & $\sqrt{ }$ \\
\hline$(20)$ & Warfarin & -3.19 & -2.94 & 1.09 & 3.10 & 70.8 & 2.28 & 2.91 & 2 & 2 & 0.62 & $\sqrt{ }$ \\
\hline (20) & Cyclosporine & -5.80 & -5.32 & 1.09 & 3.00 & 179.0 & 9.65 & 10.02 & 2 & 2 & 0.98 & $\checkmark$ \\
\hline
\end{tabular}




\begin{tabular}{|c|c|c|c|c|c|c|c|c|c|c|c|c|}
\hline \multirow[b]{2}{*}{ Ref } & \multirow[b]{2}{*}{ Drug } & \multicolumn{3}{|c|}{ Solubility (log S) } & \multicolumn{4}{|c|}{ Molecular Descriptors } & \multicolumn{2}{|c|}{ SR Group* } & \multirow[b]{2}{*}{$p$} & \multirow{2}{*}{$\begin{array}{l}\text { Prediction } \\
\text { result }\end{array}$} \\
\hline & & PBS (M) & FaSSIF (M) & $\mathrm{SR}^{*}$ & $\log \mathrm{D}_{6.5}$ & TPSA & $s$ & $v$ & Exp & Calc & & \\
\hline (20) & Indinavir & -3.90 & -4.31 & 0.90 & 3.00 & 72.5 & 4.27 & 4.90 & 2 & 2 & 0.86 & $\sqrt{ }$ \\
\hline (20) & Tolfenamic & -3.98 & -3.62 & 1.10 & 2.90 & 129.0 & 1.64 & 1.90 & 2 & 2 & 0.51 & $\sqrt{ }$ \\
\hline$(20)$ & Diazepam & -3.91 & -3.64 & 1.07 & 2.90 & 105.0 & 1.72 & 2.07 & 2 & 2 & 0.57 & $\checkmark$ \\
\hline (20) & Cilostazole & -4.77 & -4.76 & 1.00 & 2.80 & 139.0 & 2.44 & 2.85 & 2 & 2 & 0.56 & $\checkmark$ \\
\hline (20) & Rofecoxib & -4.61 & -4.53 & 1.02 & 2.70 & 119.0 & 2.43 & 2.23 & 2 & 2 & 0.83 & $\sqrt{ }$ \\
\hline (20) & Carbamazepine & -3.27 & -3.00 & 1.09 & 2.60 & 73.5 & 2.06 & 1.81 & 2 & 2 & 0.90 & $\sqrt{ }$ \\
\hline (20) & Cisapride & -5.27 & -4.86 & 1.08 & 2.60 & 182.0 & 3.15 & 3.40 & 2 & 2 & 0.63 & $\sqrt{ }$ \\
\hline (20) & Griseofulvin & -4.38 & -4.18 & 1.05 & 2.50 & 135.0 & 2.32 & 2.39 & 2 & 2 & 0.74 & $\sqrt{ }$ \\
\hline (20) & Panadiplon & -3.64 & -3.60 & 1.01 & 2.50 & 57.5 & 2.82 & 2.37 & 2 & 2 & 0.97 & $\checkmark$ \\
\hline (20) & Carvedilol & -3.95 & -3.86 & 1.02 & 2.40 & 70.9 & 3.00 & 3.10 & 2 & 2 & 0.92 & $\checkmark$ \\
\hline (20) & Amitriptyline & -2.49 & -2.50 & 1.00 & 2.40 & 2.2 & 1.31 & 2.40 & 2 & 2 & 0.68 & $\checkmark$ \\
\hline (20) & Nimesulide & -4.13 & -3.93 & 1.05 & 2.30 & 164.0 & 2.68 & 2.08 & 2 & 2 & 0.90 & $\sqrt{ }$ \\
\hline (20) & Diclofenac & -2.78 & -2.59 & 1.07 & 2.20 & 169.0 & 1.95 & 2.03 & 2 & 2 & 0.64 & $\checkmark$ \\
\hline (20) & Praziquantel & -3.17 & -3.08 & 1.03 & 2.20 & 55.0 & 2.42 & 2.45 & 2 & 2 & 0.93 & $\sqrt{ }$ \\
\hline (20) & Phenytoin & -3.81 & -3.77 & 1.01 & 2.20 & 113.0 & 2.04 & 1.87 & 2 & 2 & 0.87 & $\checkmark$ \\
\hline (20) & Haloperidol & -3.68 & -3.53 & 1.04 & 2.00 & 160.0 & 2.08 & 2.80 & 2 & 1 & 0.48 & $x$ \\
\hline (19) & Niflumic Acid & -2.40 & -2.45 & 0.98 & 1.96 & 62.2 & 1.42 & 1.79 & 2 & 2 & 0.82 & $\sqrt{ }$ \\
\hline (20) & Omeprazole & -3.28 & -3.10 & 1.06 & 1.90 & 76.9 & 3.18 & 2.52 & 2 & 2 & 0.99 & $\checkmark$ \\
\hline (19) & Rivaroxaban & -4.07 & -3.79 & 1.07 & 1.84 & 116.4 & 3.52 & 2.89 & 2 & 2 & 0.98 & $\checkmark$ \\
\hline (20) & Ibuprofen & -2.17 & -2.02 & 1.07 & 1.80 & 73.8 & 1.01 & 1.78 & 2 & 2 & 0.65 & $\sqrt{ }$ \\
\hline (20) & Dipyridamole & -4.90 & -4.64 & 1.06 & 1.80 & 103.0 & 2.90 & 3.87 & 2 & 2 & 0.74 & $\sqrt{ }$ \\
\hline (20) & Lansoprazole & -4.17 & -3.97 & 1.05 & 1.80 & 161.0 & 2.97 & 2.37 & 2 & 2 & 0.95 & $\sqrt{ }$ \\
\hline (20) & Amprenavir & -3.47 & -3.65 & 0.95 & 1.70 & 138.0 & 3.52 & 3.82 & 2 & 2 & 0.90 & $\sqrt{ }$ \\
\hline This work & Phenobarbital & -2.04 & -2.08 & 0.98 & 1.65 & 75.3 & 1.81 & 1.70 & 2 & 2 & 0.94 & $\checkmark$ \\
\hline (20) & Indomethacin & -3.21 & -2.91 & 1.10 & 1.50 & 185.0 & 2.49 & 2.53 & 2 & 2 & 0.81 & $\checkmark$ \\
\hline (20) & Quinidine & -2.19 & -2.16 & 1.01 & 1.50 & 39.4 & 1.66 & 2.59 & 2 & 2 & 0.82 & $\checkmark$ \\
\hline (20) & Digoxin & -4.69 & -4.66 & 1.01 & 1.40 & 241.0 & 4.46 & 5.75 & 2 & 1 & 0.47 & $x$ \\
\hline (20) & Sulfasalazine & -3.49 & -3.34 & 1.04 & 0.30 & 188.0 & 3.42 & 2.70 & 2 & 2 & 0.99 & $\checkmark$ \\
\hline (19) & Furosemide & -2.04 & -2.01 & 1.01 & 0.02 & 131.0 & 2.37 & 2.10 & 2 & 2 & 0.98 & $\checkmark$ \\
\hline (20) & Probenecid & -2.34 & -2.24 & 1.04 & 0.00 & 132.0 & 1.92 & 2.16 & 2 & 2 & 0.95 & $\checkmark$ \\
\hline This work & Enrofloxacin & -2.85 & -2.86 & 1.00 & -0.14 & 64.1 & 2.50 & 2.59 & 2 & 2 & 0.99 & $\checkmark$ \\
\hline (20) & Disopyramide & -3.24 & -3.03 & 1.07 & -0.20 & 58.7 & 2.26 & 2.91 & 2 & 2 & 0.98 & $\sqrt{ }$ \\
\hline This work & Lamotrigine & -2.92 & -2.82 & 1.04 & -0.22 & 90.7 & 2.13 & 1.65 & 2 & 2 & 0.99 & $\sqrt{ }$ \\
\hline
\end{tabular}

*SR > 1.1, FaSSIF has a considerable effect on solubility (Group 1) and SR <1.1, FaSSIF has no considerable effect on solubility (Group 2).

$\checkmark$ indicates agreement; $\boldsymbol{X}$ indicates disagreement. M: mean value in $\mathrm{g} / \mathrm{L}$; SR: solubilization ratio; PBS: phosphate buffer solution (pH 6.5), FaSSIF: fasted state simulated intestinal fluid, $\log D_{6.5}$ : distribution coefficient at $\mathrm{pH}=6.5, \mathrm{TPSA}$ : topological polar surface, $\mathrm{S}$ : dipolarity/polarizability descriptor; V: McGowan volume; p: probability; Exp: experimental; Calc: calculated.

7. Maghsoodi M.; Narimanpour, O. Improved dissolution behavior of dipyridamole formulation with precipitation inhibitor. Pharm. Sci. 2020, 26, 314-322. DOI: 10.34172/ PS.2020.15.

8. Yang B.; Wu, C.; Ji, B.; Ai, X.; Kuang, X.; Wu, M.; Sun, M.; Luo,
C.; He, Z.; Sun, J. The biorelevant concentration of Tween 80 solution is a simple alternative medium to simulated fasted state intestinal fluid. RSC Adv. 2015, 5, 104846104853. DOI: 10.1039/c5ra17674C.

9. Dressman J. B.; Vertzoni, M.; Goumas, K.; Reppas, C. 
Estimating drug solubility in the gastrointestinal tract. Adv. Drug Deliv. Rev. 2007, 59, 591-602. DOI: 10.1016/j. addr.2007.05.009.

10. Marques M. Dissolution media simulating fasted and fed states. Dissolut. Technol. 2004, 11, 16. DOI: 10.14227/ DT110204P16.

11. Fotaki N.; Vertzoni, M. Biorelevant dissolution methods and their applications in in vitroin vivo correlations for oral formulations. Open Drug Deliv. J. 2010, 4, 2-13. DOI: 10.2174/1874126601004010002.

12. Katritzky A. R.; Kuanar, M.; Slavov, S.; Hall, C. D.; Karelson, M.; Kahn, I.; Dobchev, D. A. Quantitative correlation of physical and chemical properties with chemical structure: utility for prediction. Chem. Rev. 2010, 110, 5714-5789. DOI: 10.1021/cr900238d.

13. Stepensky D. Prediction of drug disposition on the basis of its chemical structure. Clin. Pharmacokinet. 2013, 52, 415-431. DOI: 10.1007/s40262-013-0042-0.

14. Shayanfar A.; Acree, W. E., Jr.; Jouyban, A. Solubility of lamotrigine, diazepam, clonazepam, and phenobarbital in propylene glycol + water mixtures at 298.15 K. J. Chem. Eng. Data. 2009, 54, 1153-1157. DOI: 10.1021/je800931z.

15. Seedher N.; Agarwal, P. Various solvent systems for solubility enhancement of enrofloxacin. Indian J. Pharm. Sci. 2009; 71, 82-87. doi: 10.4103/0250-474X.51958

16. Foresti G. R.; Becker, N.;Silva, A.S.; Almeida, W. R.; Malesuik, M. D.; Hass, S. E.; Silva, F. E. An alternative method for the dissolution of enrofloxacin tablets. Dissolut. Technol. 2015, 22, 23-27. DOI: 10.14227/DT220115P23.

17. Mohana Raghava Srivalli K.; Lakshmi, P. K.; Balasubramaniam, J. Design of a novel bilayered gastric mucoadhesive system for localized and unidirectional release of lamotrigine. Saudi. Pharm. J. 2013, 21, 45-52. DOI: 10.1016/j.jsps.2012.01.004.

18. Papich M. G.; Martinez, M. N. Applying biopharmaceutical classification system (BCS) criteria to predict oral absorption of drugs in dogs: challenges and pitfalls. AAPS J. 2015, 17, 948-964. DOI: 10.1208/s12248-015-9743-7.

19. Takács-Novák, K.; Szőke, V.; Völgyi, G.; Horváth, P.; Ambrus, R.; Szabó-Révész, P. Biorelevant solubility of poorly soluble drugs: rivaroxaban, furosemide, papaverine and niflumic acid. J. Pharm. Biomed. Anal. 2013, 83, 279-285. DOI: 10.1016/j.jpba.2013.05.011.
20. Fagerberg J. H.; Karlsson, E.; Ulander, J.; Hanisch, G.; Bergström, C. A. S. Computational prediction of drug solubility in fasted simulated and aspirated human intestinal fluid. Pharm. Res. 2015, 32, 578-589. DOI: 10.1007/s11095-014-1487-z

21. Yazdanian, M.; Briggs, K.; Jankovsky, C.; Hawi, A. The "high solubility" definition of the current FDA Guidance on Biopharmaceutical Classification System may be too strict for acidic drugs. Pharm. Res. 2004, 21, 293-299. DOI: 10.1023/B:PHAM.0000016242.48642.71.

22. Ren Y. Y.; Zhou, L. C.; Yang, L.; Liu, P. Y.; Zhao, B. W.; Liu, $\mathrm{H}$. X. Predicting the aquatic toxicity mode of action using logistic regression and linear discriminant analysis. SAR QSAR Environ. Res. 2016, 27, 721-746. DOI: 10.1080/1062936X.2016.1229691.

23. Hosmer D. W., Jr.; Lemeshow, S.; Sturdivant, R. X. Applied Logistic Regression, 3rd ed.; Wiely, 2013.

24. Mandal, A. B., Nair, B.U., Ramaswamy, D. Determination of the critical micelle concentration of surfactants and the partition coefficient of an electrochemical probe by using cyclic voltammetry. Langmuir. 1988; 4, 736-739. DOI: 10.1021/la00081a041.

25. Alizadeh M. N.; Shayanfar, A.; Jouyban, A. Solubilization of drugs using sodium lauryl sulfate: Experimental data and modeling. J. Mol. Liq. 2018, 268, 410-414. DOI: 10.1016/j. molliq.2018.07.065.

26. Williams H. D.; Trevaskis, N. L.; Charman, S. A.; Shanker, R. M.; Charman, W. N.; Pouton, C. W.; Porter, C. J. H. Strategies to address low drug solubility in discovery and development. Pharmacol. Rev. 2013, 65, 315-499. DOI: 10.1124/pr.112.005660.

27. Van de Waterbeemd H.; Testa, B. Drug Bioavailability: Estimation of Solubility, Permeability, Absorption and Bioavailability; Wiely, 2009.

28. Prasanna S.; Doerksen, R. J. Topological polar surface area: a useful descriptor in 2D-QSAR. Curr. Med. Chem. 2009, 16, 21-41. DOI: 10.2174/092986709787002817.

29. Acree W. E., Jr.; Grubbs, L. M.; Abraham, M. H. Prediction of partition coefficients and permeability of drug molecules in biological systems with Abraham model solute descriptors derived from measured solubilities and water-to-organic solvent partition coefficients. In Toxicity and Drug Testing; Acree, W. E., Jr., Ed.; InTech Publisher, 2012, pp 91-128. 